\title{
One-dimensional solids made tractable
}

Schemes for using organic polymers as electrical conductors have been much dreamed of, but recent calculations suggest there is some way to go before one-dimensional solids are as simple as they seem.

Polyacetylene might be thought the simplest of polymers, consisting as it does simply of a chain of carbon atoms, each carrying a single hydrogen atom. And the simplest form of polyacetylene is that called trans, in which the successive carbon-carbon bonds zig-zag regularly to form a structure which necessarily (in the absence of stress) lies in a plane, as do the hydrogen bonds to each carbon, which lie alternately on one side of the skeleton and the other. But, of course, things are never as simple as they seem.

First, there is the little matter of the spacings between successive carbon atoms, which are joined by alternating single and double bonds, as are the atoms in a benzene ring. The expectation would be that all the bonds in such an array would be of equal length, as indeed they are in benzene and in the two-dimensional sheets of carbon atoms in graphite. In the argot of the chemistry textbooks, the four potentially bonding electrons on each carbon atom would be organized into three $\sigma$-orbitals (one towards each of two carbon neighbours and one towards a hydrogen) with the fourth occupying a familiar $\pi$-orbital.

More than thirty years have passed since Rudolf Peierls argued that transpolyacetylene (like other linear conjugated structures) is not necessarily that symmetrical. There are two potentially disturbing influences, of which the chief is the way that electric charge can slop about from one place to another in such a system. Physically, the explanation is that the energetic penalty in removing one $\pi$-electron from a single site is a fixed amount, but the effect is to change the bond configuration in one or other of the remaining halves of the whole molecule.

Alternatively, the energetic penalty in creating charge separations by as much as the whole length of the molecule is fixed, and (for long molecules) small compared with the statistical benefit of the accessibility of a whole new range of states. Yet another way of putting this point is to recognize that the electrons in such a structure form electronic bands which are in this case half-filled (so that transpolyacetylene molecules are electrically conducting), when charge separation amounts to the promotion of one electron into the conducting zone and the creation of a corresponding electron hole.

Peierls dealt with the disturbance of symmetry caused by the flopping about of electric charge with the help of what he called charge-density waves. Their effect is to break the expected symmetry of polyacetylene, meaning that the length of carbon-carbon bonds oscillates from one site to the next. More strictly, there is a phase transition, appropriately called the Peierls transition, from the symmetrical state to that of the poly-dimer. The short and long bonds in this alternating pattern are respectively $1.36 \AA$ and $1.46 \AA$ in length.

Ambitions to design organic conductors with predetermined properties are everywhere. The hope that there will one day be organic superconductors has not everywhere vanished. Polyacetylene is not so much a prototype of these materials, but a test-bed. It has the convenient property that it can be systematically doped, as semiconductors are, most conveniently by substituting electro-positive or electronegative groups for hydrogen atoms along the length of a polymer. Electron donors will add (negative) electric charge to the conduction bands, electron acceptors will subtract it.

The remarkable truth, made plain by measurement, is that the properties of polyacetylene are remarkably sensitive to the degree of charge transfer, or of doping. The average length of a repeating unit can change by several per cent by alternations of doping that imply charge transfers of 20 per cent of an electron charge from one carbon atom to its neighbour. Elastic properties, notably Young's modulus, are similarly sensitive not only to the degree of doping, but to whether the doping is by electron acceptor or donor groups.

The measurements are confusing, perhaps because there are not enough of them, but more probably because there is not a sufficiently well-developed framework of reasonable expectation. But people seem aware of what is needed. Two groups have recently reported attempts to calculate the properties of doped polyacetylene and such materials. Sung Y. Hong and Miklos Kertesz from Georgetown University (Washington, D.C.) appear to have gone a long way to account for recorded measurements of polyacetylene (Phys. Rev. Lett. 64, 3031; 18 June 1990).

To calculate elastic constants, the first need is to estimate how the energy surface varies in the neighbourhood of equilibrium as a function of parameters describing the structure of the molecule. Hong and Kertesz do this in two ways, by using an infinite molecular chain and one consisting of 21 carbon atoms (with the ends of the chain sealed off with extra hydrogen atoms). Both schemes rely on the computer codes which have become the bread and butter of computational chemistry. The outcome is satisfying in two respects - the predicted variations of Young's modulus with doping is not merely of the same order of magnitude as the measurements, but the qualitative variation of the modulus with the degree of doping is that found in practice, even to the extent that the asymmetry with the sense of the doping is reproduced.

Joseph A. Aronovitz, Paul Goldbart and George Mozurkewich from the University of Illinois at UrbanaChampaign set out to demonstrate a more subtle point by a more general argument (Phys. Rev. Lett 64, 2799; 4 June 1990). Their interest is not simple polyacetylene, but the mostly inorganic materials such as $\mathrm{K}_{03} \mathrm{MoO}_{3}$, which is called blue-bronze, and $\left(\mathrm{TaSe}_{4}\right)_{2} \mathrm{I}$, both of which form solid crystals consisting of parallel strings of atoms which can act as conductors and which support charge-density waves just as if they were polyacetylene. Both materials have abnormally high thermal conductivity as well as specific heat anomalies near their Peierls transitions, at $180 \mathrm{~K}$ and $263 \mathrm{~K}$ respectively.

What Aronovitz, Goldbart and Mozurkewich have done is to consider the interaction between lattice vibrations and charge-density waves, which are known to be coupled together strongly near the transition point. The argument follows the outlines of V. L. Ginzburg's treatment of phase transitions in superconducting solids, for example, but one conclusion is that the behaviour of these one-dimensional solids resembles, near the transition point, the phenomenon of magnetoelasticity.

The upshot of an intricate argument is that changes of the elastic constants near the Peierls transition should be related in a simple (and calculable) way to the anomaly in the specific heat, which on a graph of specific heat against temperature lies like a cusp above a smooth background (which shows that the entropy at the transition is finite). The further remarkable prediction is that there should be similar anomalies of the elastic constants near the transitions, which have yet to be observed. If the prediction is confirmed, who will be the first to make a device of it? And what for?

John Maddox 